\title{
Induction of inner ear hair cell-like cells from Math1-transfected mouse ES cells
}

\author{
Y Ouji ${ }^{\star, 1,2}$, S Ishizaka ${ }^{2}$, F Nakamura-Uchiyama ${ }^{1}$, A Wanaka ${ }^{3}$ and M Yoshikawa ${ }^{*, 1,2}$
}

Math1, a basic helix-loop-helix transcription factor homolog of the Drosophila atonal gene, is considered to be a key factor for induction of sensory hair cells (HCs) during development of the organ of Corti or cochlea. Although embryonic stem (ES) cells are able to produce HC-like cells, the role of Math1 in induction of those cells has not been thoroughly elucidated. In the present study, we introduced Math1 into ES cells in order to achieve efficient generation of HC-like cells. ES cells carrying Tet-inducible Math1, Math1-ES cells, were generated using a Tet-On gene expression system. Embryoid bodies (EBs) formed in the absence of doxycycline (Dox) for 4 days were allowed to grow for an additional 14 days in the dishes in the presence of $400 \mu \mathrm{g} / \mathrm{ml}$ of Dox. At the end of those 14-day cultures, approximately $10 \%$ of the cells in EB outgrowths expressed the HC-related markers myosin6, myosin7a, calretinin, $\alpha 9 A c h R$, and Brn3c (also known as Pou4f3) and showed formation of stereocilia-like structures, whereas few cells in EB outgrowths grown without Dox showed those markers. Reporter assays of Math1-ES cells using a Brn3cpromoter plasmid demonstrated positive regulation of Brn3c by Math1. Furthermore, such HC-related marker-positive cells derived from Math1-ES cells were found to be incorporated in the developing inner ear after transplantation into chick embryos. Math1-ES cells are considered to be an efficient source of ES-derived HC-like cells, and Math1 may be an important factor for induction of $\mathrm{HC}$-like cells from differentiating ES cells.

Cell Death and Disease (2013) 4, e700; doi:10.1038/cddis.2013.230; published online 4 July 2013

Subject Category: Experimental Medicine

The mammalian inner ear contains the cochlea and vestibular organs, which are responsible for hearing and balance, respectively. In the mammalian auditory system, sensory hair cells (HCs) in the organ of Corti do not replace lost HCs, ${ }^{1,2}$ though regeneration of $\mathrm{HCs}$ is well known in the avian cochlea. ${ }^{3-5}$ Embryonic stem (ES) cells and induced-pluripotent stem cells are particularly promising candidates for cell replacement therapy, because of their pluripotency for differentiation and limitless capability for reproduction in vitro. ${ }^{6-8}$

Recently, molecular mechanisms related to $\mathrm{HC}$ development have been elucidated in detail, ${ }^{9-12}$ while the transcription factor Math1 (also known as Atoh1) has been identified as the earliest $\mathrm{HC}$-specific gene required for definitive $\mathrm{HC}$ development. ${ }^{13,14}$ Loss of Math1 results in complete failure of $\mathrm{HC}$ differentiation in the mouse cochlea, ${ }^{15}$ while its overexpression via viral vectors was reported to generate new $\mathrm{HCs}^{16,17}$ and substantially improve hearing thresholds in adult deaf guinea pigs. ${ }^{18}$ Furthermore, it has also been reported that introduction of Math1 led to successful generation of HC-like cells from bone marrow mesenchymal stem cells. ${ }^{19}$ Thus, Math 1 seems to be a key transcription factor for $\mathrm{HC}$ development. In addition, Math1 is known as a master gene for cerebellar granule neuron (CGN) differentiation, ${ }^{20}$ and its temporally controlled transient expression was recently reported to drive ES cells toward a cerebellar fate. ${ }^{21}$ However, its effects on $\mathrm{HC}$ differentiation of ES cells have not been reported. In the present study, we investigated the effects of Math1 overexpression on HC differentiation of ES cells.

We introduced Math1 into ES cells under regulation by a Tet-On gene expression system. ${ }^{22}$ Using ES cells carrying Tet-inducible Math1, embryoid bodies (EBs) grown for 4 days without doxycycline (Dox) were then cultured for 14 days in the presence of Dox. At the end of the cultures, approximately $10 \%$ of the cells in EB outgrowths expressed HC-related markers, including myosin6, myosin7a, calretinin, $\alpha 9 A c h R$, and Brn3c (also known as Pou4f3) and showed formation of stereocilia-like structures. Reporter assays of Math1-ES cells using a Brn3c-promoter plasmid demonstrated positive regulation of $B r n 3 c$ by Math1. Furthermore, we also found that such HC-related marker-positive cells derived from Math1-ES cells were incorporated in the developing inner ear after transplantation into chick embryos. Thus, Math1-ES cells are considered to be an efficient source of ES-derived HC-like cells, and Math1 is an important factor for induction of $\mathrm{HC}$-like cells from differentiating ES cells.

\section{Results}

Establishment of ES cells expressing Tet-inducible Math1 (Math1-ES cells). ES cells were transfected with pTet-On and neo-resistant clones were obtained. In order to determine the inducing ability of rtTA protein, they were

\footnotetext{
${ }^{1}$ Department of Pathogen, Infection and Immunity, Nara Medical University, Kashihara, Nara, Japan; ${ }^{2}$ Program in Tissue Engineering and Regeneration Medicine, Nara Medical University, Kashihara, Nara, Japan and ${ }^{3}$ Department of Anatomy and Cell Biology, Nara Medical University, Kashihara, Nara, Japan

*Corresponding author: Y Ouji or M Yoshikawa, Department of Pathogen, Infection and Immunity, Nara Medical University, 840 Shijo-cho, Kashihara, Nara 634-8521, Japan. Tel: +81 74429 8847; Fax: +81 74429 8847; E-mail: oujix@naramed-u.ac.jp (YO) or myoshika@naramed-u.ac.jp (MY)

Keywords: Math1; embryonic stem cells; inner ear; hair cells; differentiation; Tet-On system

Abbreviations: HC, hair cell; ES, embryonic stem; Dox, doxycycline; EB, embryoid bodies

Received 26.11.12; revised 10.5.13; accepted 03.6.13; Edited by Y Shi
} 
transiently transfected with the reporter gene plasmid pTRE2hyg-Luc. Of the 24 clones examined, no. 14 showed the highest luciferase activity in the presence of Dox and was selected and named Tet-ES (Supplementary Information, Supplementary Figure S1C). Tet-ES cells were transfected with pTRE2pur and pTRE2pur-Math1. Puromycin-resistant cells were cloned and screened for Math1 gene expressions by RT-PCR after 2-day cultures in the presence of $1 \mu \mathrm{g} / \mathrm{ml}$ Dox (Supplementary Information, Supplementary Figure S1D). Ten of the 16 clones were found to express Math1. Four of those, nos. 1, 3, 5, and 6, were selected and named Math1-ES1, Math1-ES3, Math1-ES5, and Math1ES6, respectively. Each Math-ES clone demonstrated similar results in the following experiments, thus representative results from only Math1-ES1 are presented.

Verification of Math1-ES cells. We first examined whether Math1-ES cells possessed characteristics of undifferentiated ES cells. Math1-ES cells cultured in ES medium without Dox formed colonies similar to those of undifferentiated WT-ES cells and were also immune-positive for ALP (Figure 1a). Furthermore, RT-PCR findings confirmed that they expressed markers of an undifferentiated state, such as Oct3/4 and UTF1 (Figure 1b), suggesting that such a state was maintained when Math1-ES cells were cultured in ES medium without Dox.

Next, we examined the expression of Math 1 in the presence of Dox. The level of mRNA for Math1 after $48 \mathrm{~h}$ of exposure to Dox was tightly regulated in a dose-dependent manner and reached a plateau at a concentration $>1 \mu \mathrm{g} / \mathrm{ml}$, whereas no expression was observed in the absence of Dox (Figure 1c). In the presence of $1 \mu \mathrm{g} / \mathrm{ml}$ Dox, mRNA became detectable within $12 \mathrm{~h}$ and was drastically increased at $48 \mathrm{~h}$ (Figure 1d). Immunocytochemical analysis also demonstrated that Math1-ES cells were immune-positive for Math1 proteins after a 48 -h culture in the presence of $1 \mu \mathrm{g} / \mathrm{ml}$ Dox but not in its absence (Figure 1e).

Deviated differentiation of EBs toward ectoderms. Math1-ES-derived EBs were formed using a hanging drop method with ES medium without LIF in the absence of Dox for 4 days, then the resultant 4-day EBs were allowed to grow in dishes in the presence or absence or Dox for 2 weeks (Figure 2a). The appearance of EB outgrowths cultured in the absence of Dox was rather heterogeneous and often included beating cells (Figure 2b, $\operatorname{Dox}(-)$ arrows, Supplementary Information, Supplementary Movie S1), as observed in previous experiments. ${ }^{23}$ On the other hand, EB outgrowths cultured in the presence of Dox showed a less heterogeneous appearance and beating cells did not appear (Figure 2b, Dox $(+)$ ). During the 2-week cultivation, the number of beating focuses in culture dishes with medium lacking Dox reached a maximum by day 7 and then decreased, while no beating focuses appeared in culture dishes with the medium containing Dox throughout the experimental period (Figure 2c).

We also examined the mRNA expressions of nestin and $N F-L$ as neuroectoderm markers, ${ }^{24} \zeta$-globin and Nkx2.5 as mesoderm markers, ${ }^{25}$ and AFP and TTR as endoderm markers $^{26}$ (Figure 2d). Nestin and NF-L were firmly expressed as early as day 2 in EB outgrowths cultured in the presence of Dox, while they did not become detectable until after day 2 in those cultured in its absence. In contrast, no expressions of $\zeta$-globin or Nkx2.5 were observed throughout the 2-week period in Dox-treated EB outgrowths, while they were detected on days 2, 7, and 14 in Dox-untreated EB outgrowths. Immunocytochemical analyses also revealed immune-positive cells for nestin and NF-L but not for Nkx2.5 in Dox-treated EB outgrowths (Supplementary Information, Supplementary Figure S2). AFP and TTR were expressed on day 14 in Dox-untreated EB outgrowths but not in Dox-treated EB outgrowths. These results indicate that forced expression of Math1 results in preferentially deviated differentiation in an ectodermal direction.

Expression analysis of HC-related markers in the EB outgrowths. The gene expressions of Math1, myosin6, myosin7a, Brn3c, and $\alpha 9 A c h R$ during the 2-week cultivation of Math1-ES-derived EBs were analyzed using a semiquantitative RT-PCR method (Figure 3a). Math1 was clearly detected in all the samples from EB outgrowths cultured in the presence of Dox but not in those cultured in its absence. All other HC-related markers, including myosin6, myosin7a, $B r n 3 c$, and $\alpha 9 A c h R$, were also detected by the end of the 2-week culture in the presence of Dox, whereas none were detected in its absence, except for a trace expression of myosin7a. Immunocytochemical analyses of myosin6, Brn3c, and $\alpha 9 A c h R$ demonstrated their expressions in EB outgrowths cultured in the presence of Dox for 2 weeks (Figure 3b). The percentages of cells immunopositive for the investigated markers in the EB outgrowths cultured with Dox were as follows: myosin6, $12.8 \pm 1.2 \%$; Brn3c, $9.8 \pm 1.3 \%$; and $\alpha 9$ AchR, $8.9 \pm 0.8 \%$, while those in cultures without Dox were $1.5 \pm 0.1 \%, 1.0 \pm 0.2 \%$, and $0.8 \pm 0.2 \%$, respectively (Figure $3 c$ ). Furthermore, $87 \pm 2.5 \%$ of myosin6-immunopositive cells were positive for Brn3c, $89 \pm 3.5 \%$ of $\alpha 9$ AchRimmunopositive cells were positive for myosin6, and $89 \pm 2.8 \%$ of Brn3c-immunopositive cells were positive for $\alpha 9 A c h R$ (Figure 3d). These results indicate that myosin6, Brn3c, and $\alpha 9 A c h R$ were simultaneously expressed at extremely high levels.

Immunocytochemical analysis of HC-related markers in the context of Math1 expression. In EB outgrowths with or without Dox treatment, immunocytochemical expressions of myosin6, Brn3c, and $\alpha 9 A c h R$ were investigated in the context of Math1 expression. Although all cells in outgrowths cultured in the presence of Dox were immunopositive for Math1 under control of the Tet-On system, some were intensely positive and easily distinguished from the other vaguely positive cells, as shown in a modified observation (Figure $4 \mathrm{a}$, Math1 M). Interestingly, $>80 \%$ of the cells intensely positive for Math1 were also immune-positive for myosin6 (90.2 $\pm 3.5 \%)$, Brn3c $(82.4 \pm 4.2 \%)$, and $\alpha 9 A c h R$ $(88.5 \pm 4.9 \%)$ (Figure $4 b)$. In contrast, there were few Math1immunopositive cells generated in outgrowths cultured without Dox and those were rarely immunopositive for myosin6, Brn3c, or $\alpha 9 A c h R$. These results strongly suggest that forced expression of Math1 preferentially promotes generation of cells expressing $\mathrm{HC}$-related markers in EB outgrowths. 
a
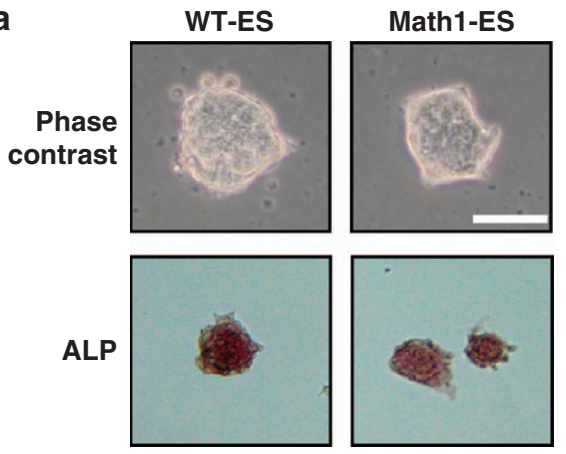

b

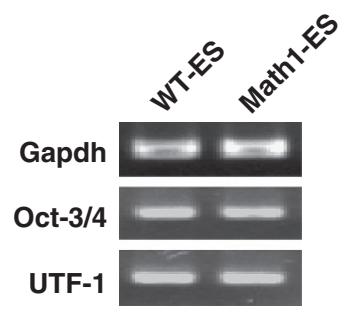

C

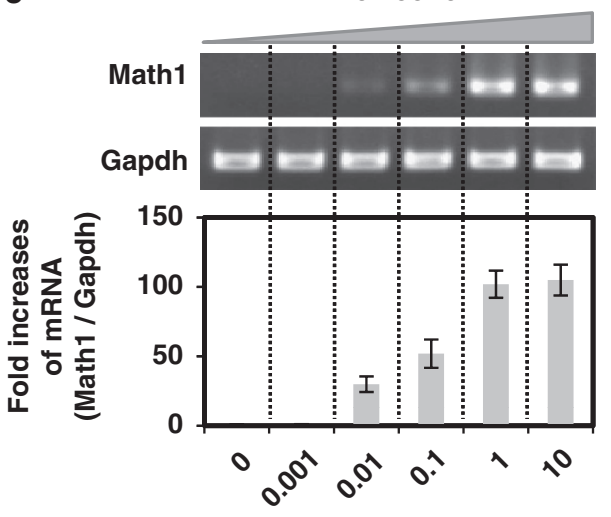

Dox $[\mu \mathrm{g} / \mathrm{ml}]$ d

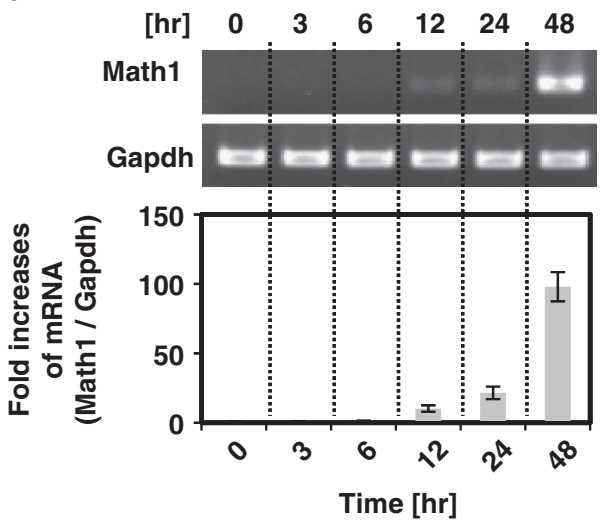

e
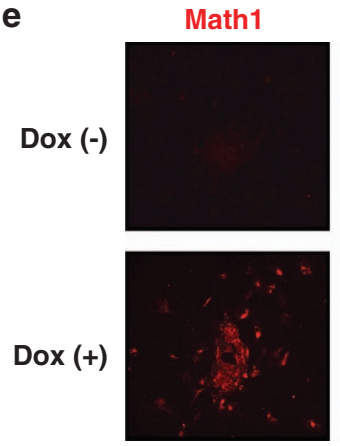

DAPI
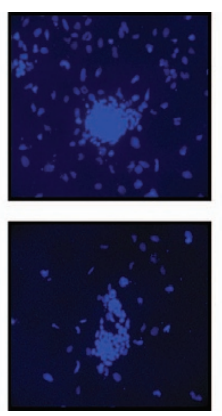

Merge
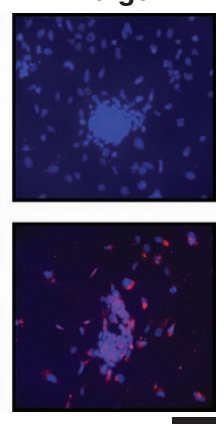

Figure 1 Verification of Math1-ES cells. (a) Math1-ES cells cultured in ES medium without Dox formed colonies similar to those of undifferentiated wild type ES cells (WT-ES cells). Math1-ES and WT-ES cells were immune-positive for alkaline phosphatase (ALP). Bar $=50 \mu \mathrm{m}$. (b) RT-PCR findings showed that Math1-ES cells cultured in ES medium without Dox expressed Oct3/4 and UTF-1. (c) The level of Math $1 \mathrm{mRNA}$ after $48 \mathrm{~h}$ of exposure to Dox was tightly regulated in a dose-dependent manner. (d) The level of Math1 mRNA became detectable within $12 \mathrm{~h}$ and was drastically increased after $48 \mathrm{~h}$ in the presence of $1 \mu \mathrm{g} / \mathrm{ml}$ Dox. (e) Math1-ES cells were immunopositive for Math1 after a 48-h culture in the presence of $1 \mu \mathrm{g} / \mathrm{ml}$ Dox. Bar $=20 \mu \mathrm{m}$. GAPDH, glyceraldehyde 3-phosphate dehydrogenase; DAPI, 4,6-diamidino-2-phenylindole

Mechanotransduction channels and stereocilia-like structures in HC-like cells. EB outgrowths cultured with and without Dox for 2 weeks were examined using phalloidinstaining, FM1-43FX dye, and a scanning electron microscope (SEM). Phalloidin staining and FM1-43FX dye enable determination of actin-rich hair bundles of $\mathrm{HCs}^{27}$ and identification of mechanotransduction channels in $\mathrm{HCs},{ }^{28,29}$ respectively. Phalloidin-positive cells were found in EB outgrowths treated with Dox (Figure 5A), while these were not observed in outgrowths without Dox. Furthermore, a marked number of FM1-43FX-positive cells $(8.3 \pm 1.4 \%)$ were identified in outgrowths treated with Dox (Figure 5B and Table 1), whereas very few $(0.41 \pm 0.3 \%)$ were detected in those without Dox. In addition, immunostaining with Brn3c following FM1-43FX dye treatment and fixation demonstrated that nearly all FM1-43FXpositive cells were Brn3c-immunopositive (Table 1), indicating the emergence of $\mathrm{HC}$-like cells with potentially functional mechanotransduction channels. Also, cells with distinct stereocilia-like structures were observed in EB outgrowths grown with Dox by $\mathrm{SEM}^{30}$ (Figure $5 \mathrm{Cc}$, Figure $5 \mathrm{Cd}$ ), while cells with such structures were not found among those grown without Dox (Figures 5Ca and 5Cb). 
a

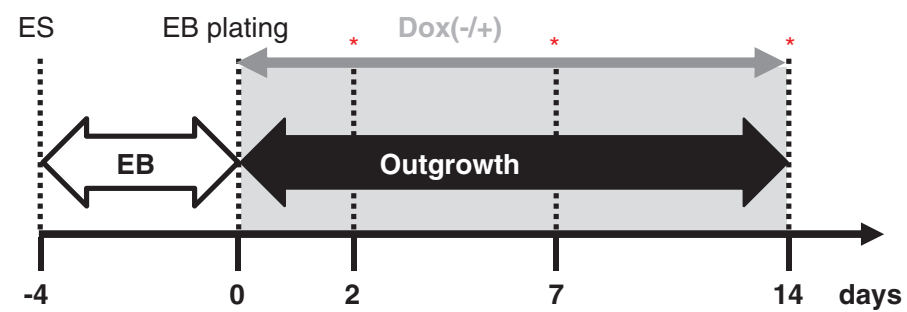

b

2d
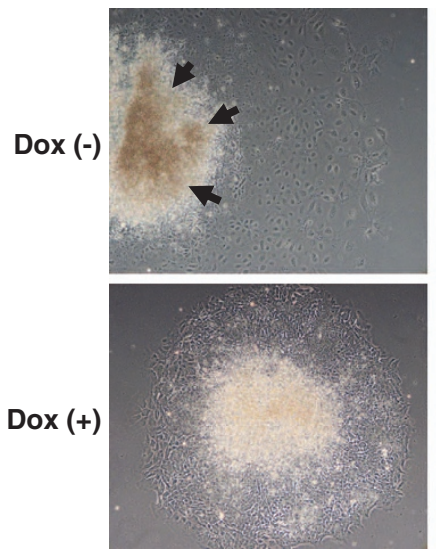

C

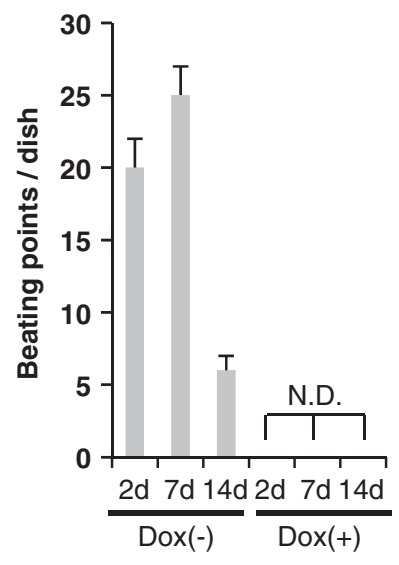

$7 d$
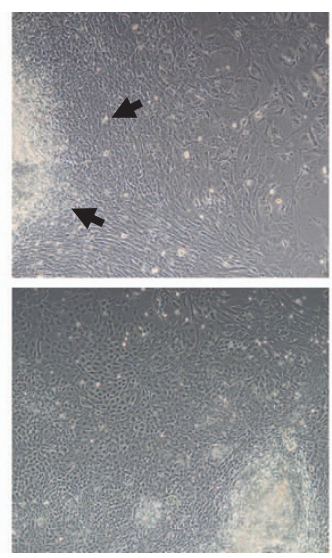

d

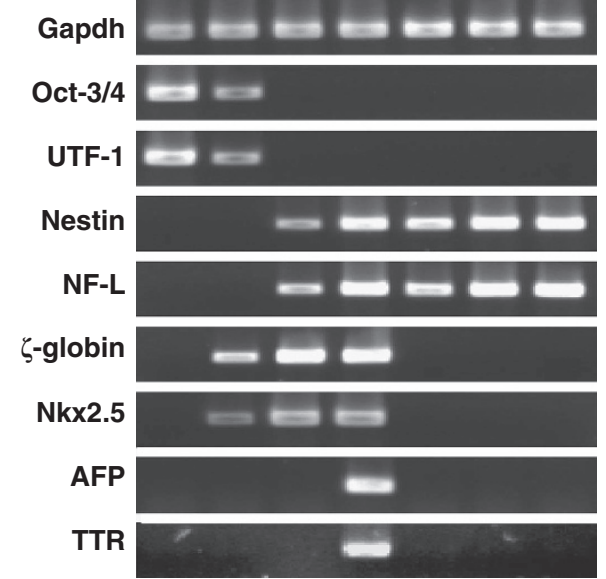

Figure 2 EB differentiation deviated toward ectoderm. (a) Experimental protocol used for differentiation. After EB formation for 4 days, Math1-ES-derived EBs were cultured with or without Dox for 2 weeks. (b) Photographs of EB outgrowths after 2 weeks of cultivation. Arrows indicate beating cells. Bar $=100 \mu \mathrm{m}$. (c) Numbers of beating focuses with and without 2 weeks of exposure to Dox. (d) Analysis of expressions of non-differentiation markers (Oct-3/4, UTF-1), ectodermal markers (nestin, NF-L), mesodermal markers ( $\zeta$-globin, Nkx2.5), and endodermal markers (AFP, TTR) in EB outgrowths after cultivation with or without Dox for 2 weeks. GAPDH, glyceraldehyde 3-phosphate dehydrogenase; N.D., not detected

\begin{abstract}
Math1-dependent induction of HC-like cells from ES cells. We recently developed a simple and efficient technique to obtain ES-derived $\mathrm{HC}$-like cells with a relatively short period of cultivation, termed the HIST2 method, in which only conditioned medium from cultured ST2 stromal cells is used. $^{31}$ In the present study, we examined whether induction of $\mathrm{HC}$-like cells from ES cells occurred in a Math1-dependent or -independent manner using siRNA. Suppression of Math1 by a specific siRNA led to inhibition of $B r n 3 c$ expression and a decreased number of Brn3c-immunopositive cells in EB
\end{abstract}

outgrowths grown in ST2-conditioned medium (Figures 6a and $b$ ), indicating that the induction of $\mathrm{HC}$-like cells from ES cells with the HIST2 method was a Math1-dependent process. Furthermore, we performed reporter assays of Math1-ES cells using a Brn3c-promoter plasmid, which confirmed positive regulation of Brn3c by Math1 in both undifferentiated ES cells and EB outgrowths (Figure 6c). Based on results of our siRNA experiments and reporter assays, the induction of $\mathrm{HC}$-like cells from ES cells was considered to be achieved via Math1-driven differentiation. 
a
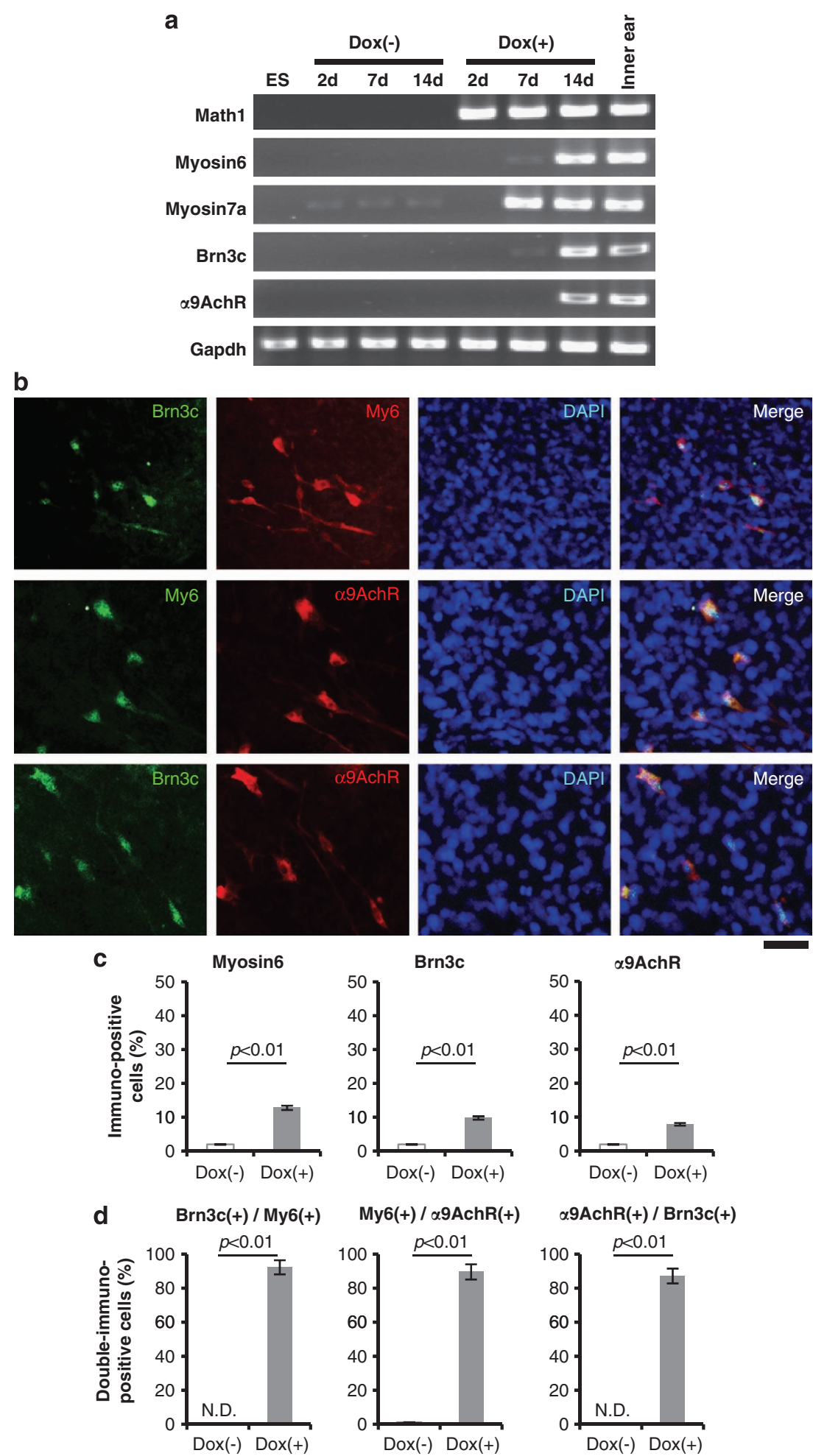

Figure 3 Analysis of expressions of $\mathrm{HC}$-related markers in Math1-ES-derived EB outgrowths. (a) Analysis of expressions of Math1, as well as myosin6, myosin7a, Brn3c, and $\alpha 9 A c h R$ as $\mathrm{HC}$-related markers in EBs cultured with or without Dox for 2 weeks. (b) Immunocytochemical analyses of myosin6 (My6), Brn3c, and $\alpha 9 A c h R$ in EB outgrowths cultured in the presence of Dox for 2 weeks. Bar $=50 \mu \mathrm{m}$. (c) Frequencies of immunopositive cells for My6, Brn3c, and $\alpha 9 A$ chR in EB outgrowths cultured for 2 weeks with or without Dox $(n=3)$. Without Dox, immunopositivity for myosin6, Brn3c, and $\alpha 9 A c h R$ was seen at very low levels. In contrast, a remarkably increased number of immunopositive cells was detected in EB outgrowths cultured with Dox. (d) Simultaneous expressions of My6, Brn3c, and $\alpha 9 A$ chR in EB outgrowths cultured with or without Dox $(n=3)$. In cultures with Dox, the three markers were simultaneously expressed at high frequencies. GAPDH, glyceraldehyde 3-phosphate dehydrogenase; DAPI, 4,6-diamidino-2-phenylindole 

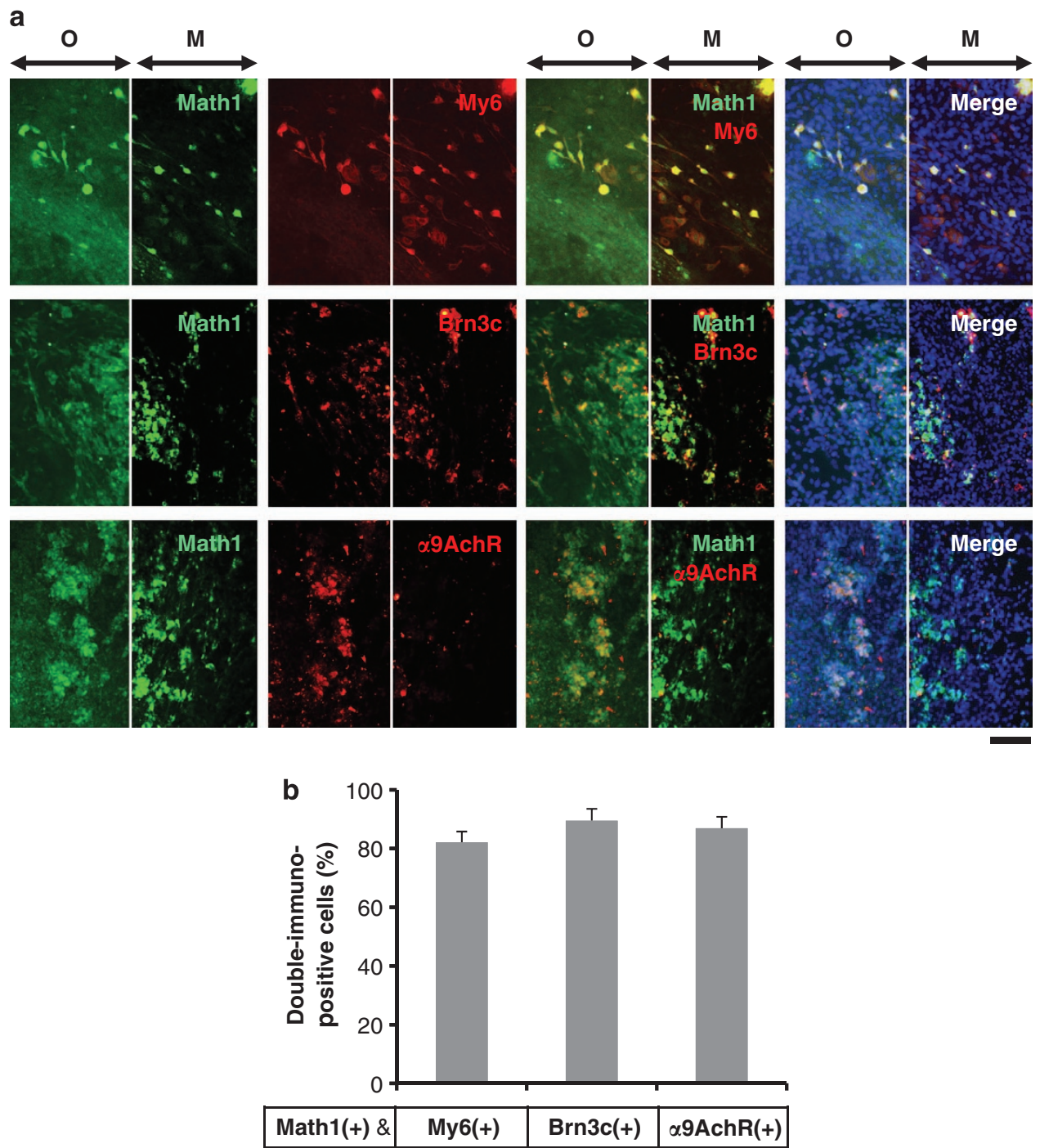

Figure 4 Immunocytochemical analysis of HC-related markers in the context of Math1 expression. (a) Math1-ES-derived EB outgrowths were treated with Dox for 2 weeks, then examined for expressions of myosin6, Brn3c, and $\alpha 9 A c h R$ in the context of Math1 expression. Cells intensively positive for Math1 were easily distinguished from other vaguely positive cells in visual observations $(\mathrm{O})$ and also selectively evident in modified observations $(\mathrm{M})$. $\mathrm{Bar}=50 \mu \mathrm{m}$. (b) Using an immunocytochemical method, the expressions of My6, Brn3c, and $\alpha 9 A$ chR were investigated among cells intensively positive for Math1 in Math1-ES-derived EB outgrowths treated with Dox for 2 weeks. The three markers were simultaneously expressed at high frequencies

Transplantation of cells from Math1-ES-derived EB outgrowths into chick embryos. To examine whether $\mathrm{HC}$-like cells are integrated in living animals after injection, we implanted cells from Math1-ES-derived EB outgrowths cultured with Dox for 2 weeks into the otic vesicles of chicken embryos. Three days after injection, morphological analyses were performed (Figure 7), and the cochlear duct, lateral ampulla, and semicircular canal were confirmed (Figures $7 \mathrm{a}$ and b). Furthermore, Math1-ES-derived cells, identified by labelling with the cell tracer, were found to be integrated in an orthotopic manner and expressed HC-related markers, such as myosin6 and Brn3c (Figures 7c and d).

\section{Discussion}

The molecular mechanisms underlying $\mathrm{HC}$ development and differentiation are poorly understood. Mice with targeted deletion of the transcription factor Brn3c (Pou4f3) begin to lose HCs by embryonic day 17 and are deaf from birth. ${ }^{32}$ Similarly, embryonic Math1-null mice fail to generate cochlear and vestibular HCs. ${ }^{15}$ Thus, these genes are required for the genesis of $\mathrm{HCs}$. It has been reported that transfer of adenoviral vectors expressing Math1 into the ears of guinea pigs resulted in formation of $\mathrm{HC}$-like cells. ${ }^{17}$ Furthermore, Math1 was found to induce regeneration of $\mathrm{HCs}$ and improve hearing thresholds after delivery to non-sensory cells through adeno-vectors, ${ }^{18}$ and it is also known that overexpression of Math1 causes differentiation of bone marrow mesenchymal stem cells into HC-like cells. ${ }^{19}$ Thus, Math1 is considered to be essential for differentiation and regeneration of $\mathrm{HCs}$ and also important for genesis of HC-like cells from other cell types.

We established Math1-introduced ES cells (Math1-ES cells), in which the expression of exogenously transferred Math1 was controlled using a Tet-On system. After 
A
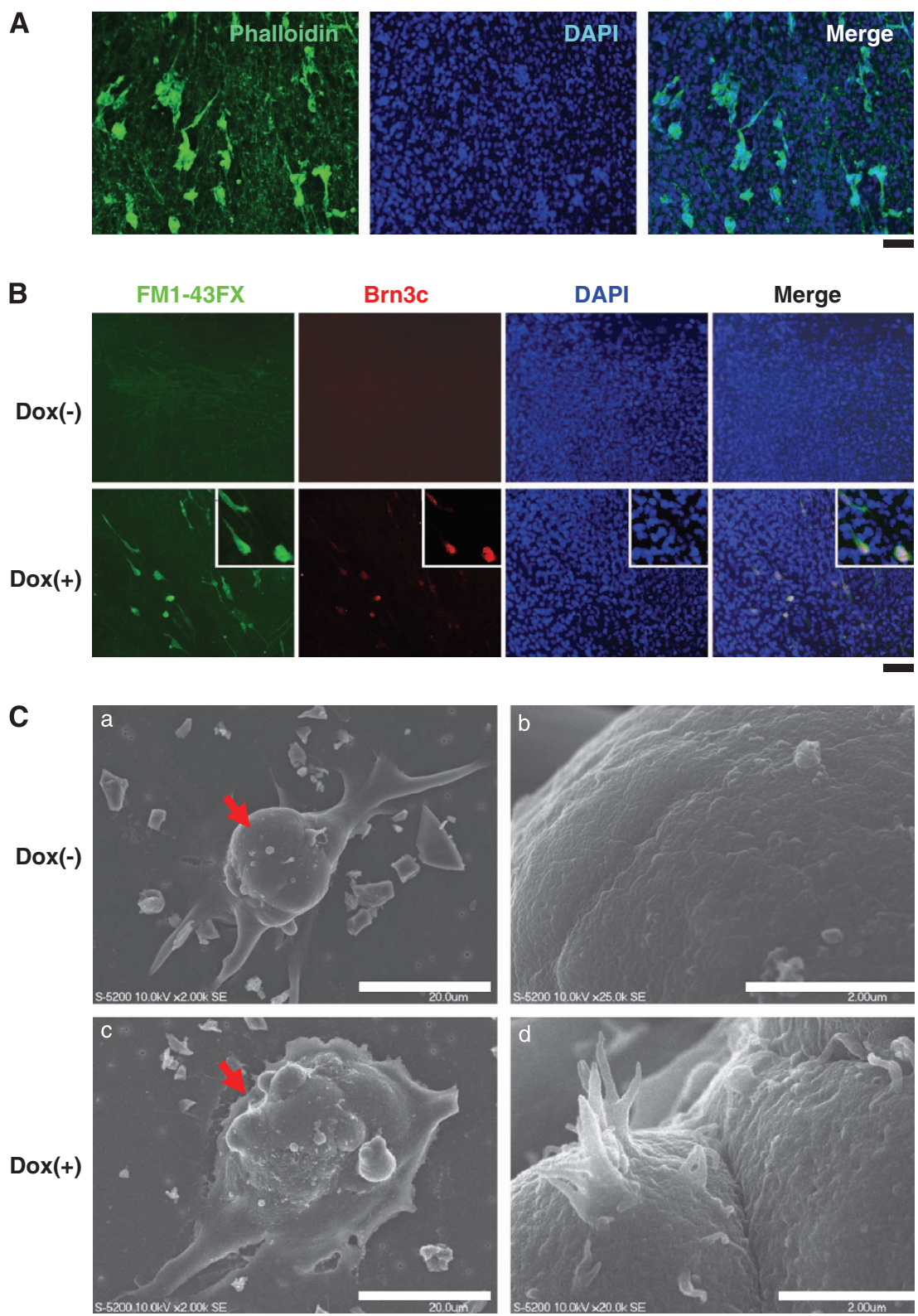

Figure 5 Mechanotransduction channels and stereocilia-like structures in HC-like cells. (A) Phalloidin-positive cells were found in Math1-ES-derived EB outgrowths treated with Dox. Bar $=40 \mu \mathrm{m}$. (B) Math1-ES-derived EB outgrowths cultured with Dox were treated with FM1-43FX dye and stained with Brn3c after fixation. A marked number of FM1-43FX-positive cells were identified in the EB outgrowths. Insets: high magnification views. Bar $=50 \mu \mathrm{m}$. (C) Math1-ES-derived EB outgrowths cultured with and without Dox for 2 weeks were examined using a SEM. (c and d) Cells with distinct stereocilia-like structures were observed in the EB outgrowths cultured with Dox, while $(a$ and $b)$ no cells presenting such structures were found among those cultured without Dox. ( $b$ and d): High magnification views of the areas in panels ( $a$ and $c$ ), respectively (red arrows). Bars $=20 \mu \mathrm{m}$ (a and $c$ ) and $2 \mu \mathrm{m}$ (b and d)

experimenting with several different protocols, including direct exposure to Dox of undifferentiated single ES cells or EB formations to efficiently yield ES-derived HC-like cells, the current protocol was adopted (Figure 2a). Interestingly, the differentiation of Math1-ES cells was strongly deviated to a neuroectodermal direction in the presence of Dox and resulted in the promotion of expression of HC-related markers. Cells with HC-related markers possessed the potential of functional mechanotransduction channels. In addition, formation of stereocilia-like structures, a characteristic morphology for $\mathrm{HCs}$, was observed in EB outgrowths treated with Dox. Furthermore, Math1-dependent induction of $\mathrm{HC}$-like cells from ES cells was confirmed in the present siRNA experiment and positive regulation of $B r n 3 c$ by Math 1 in findings of reporter assays using a Brn3c-promoter plasmid. Taken together, induction of HC-like cells from ES cells was considered to be achieved via Math1-driven differentiation.

Others have provided supporting evidence showing Math1driven differentiation toward a certain direction. Math1 is also known as a master gene in the differentiation of $\mathrm{CGNs}^{20}$ It was recently reported that temporally controlled transient expression of Math 1 drove ES cells toward a cerebellar fate. ${ }^{21}$ We 
Table 1 FM1-43FX uptake in hair cell-like cells

\begin{tabular}{lrr}
\hline & Dox(-) & Dox(+) \\
\hline FM1-43FX $(+)(\%)^{a}$ & $0.41 \pm 0.3$ & $8.3 \pm 1.4$ \\
Brn3c $(+)(\%)^{b}$ & $1.26 \pm 0.3$ & $9.6 \pm 1.9$ \\
FM1-43FX $(+) / \operatorname{Brn3c}(+)(\%)^{c}$ & $24.5 \pm 0.9$ & $87.5 \pm 2.9$
\end{tabular}

aThe percentages were quantified using FM1-43FX-positive cells and nuclei stained by DAPI $(n=15)$.

${ }^{b}$ The percentages were quantified using Brn3c-immunopositive cells and nuclei stained by DAPI $(n=15)$.

${ }^{\circ}$ Brn3c immunostaining was performed after FM1-43FX dye treatment and fixation. The percentages of FM1-43FX-positive cells in Brn3c-immunopositive cells are shown $(n=15)$.

examined the expressions of CGN markers (PDE1C and GABAa6R) and found the cells in Math1-ES-derived EB outgrowths treated with Dox to be immunopositive for PDE1C $(6.2 \pm 1.2 \%)$ and GABAa6R $(9.8 \pm 1.1 \%)$, whereas none or scant numbers of such immunopositive cells were seen in the outgrowths without Dox (Supplementary Information, Supplementary Figure S3).

In the present study, some cells in Math1-ES-derived EB outgrowths treated with Dox were intensely immunopositive for Math1 (Figure 4a, Math1 M). Interestingly, those intensively positive cells, but not vaguely positive cells, were also immunepositive for the HC-related markers myosin6, Brn3c, and $\alpha 9 A c h R$. As the expression of $B r n 3 c$ is Math1 dependent, it is conceivable that markers of $\mathrm{HC}$ cells were expressed in a restricted manner in cells intensively positive for Math1. However, we did not find strong differences in Math1immunopositivity in ES-derived EB outgrowths treated with the HIST2 method. Although the reason for the difference in degree of Math1-immunopositivity has not been determined from our findings, it might have been due to the additive effect of endogenous Math1 expression. Transcribed signaling from endogenous Math1 was detected in the Math1-ES-derived EB outgrowths cultured with Dox for $>1$ week (Supplementary Information, Supplementary Figure S4). Thus, the emergence of endogenous Math1 expression suggests positive autoregulation mechanisms in Math1 expression, as previously reported. ${ }^{33,34}$

Recently, we developed a simple and efficient technique, termed the HIST2 method, to obtain ES-derived HC-like cells with a relatively short period of cultivation, in which only conditioned medium from cultured ST2 stromal cells is used. We examined whether more effective and efficient induction of HC-like cells from ES cells can be achieved by combining Math1 gene regulation by the Tet-On system with our HIST2 method. As expected, Brn3c-immunopositive cells appeared at the highest frequency $(28.4 \pm 6.2 \%)$ in Math1-ES-derived EB outgrowths treated with Dox when using the HIST2 method, as compared with other conditions (Supplementary Information, Supplementary Figure S5), indicating that humoral factors were present in the ST2 conditioned medium. Even though the components of this natural cocktail remain to be chemically defined, it is possible that it can potentiate the development of HC-like cells from Math1-ES cells. We consider that the use of unknown extrinsic growth factors in combination with Math1 gene regulation provides more efficient induction of HC-like cells from ES cells. On the other hand, more precise Math1 control may be required to obtain not only HC-like cells but also supporting cells such as SC-like cells, because even though Math1 expression is continuously maintained in $\mathrm{HCs}$, it is downregulated in SCs in the developing cochlea after the formation of pro-sensory epithelium, which expresses Math $1{ }^{34}$

In conclusion, we investigated the differentiation of ES cells into inner ear HCs using Math1 gene transfer and a Tet-On regulation system. ES cells carrying Tet-inducible Math1 showed a high ability to differentiate into HC-like cells in EBattached cultures in the presence of Dox, as approximately $10 \%$ of the cells were differentiated after a 2-week exposure to Dox. Furthermore, we found that these cells were incorporated in the developing inner ear after transplantation into chick embryos. Thus, Math1 is an important factor for induction of HC-like cells into differentiating ES cells, and Math1-ES cells are an efficient source to obtain ES-derived HC-like cells.

\section{Materials and Methods}

Construction of vectors. A Tet-On gene expression system (BD Bioscience, Bedford, MA, USA) was used for Dox-dependent gene regulation. A plasmid containing mouse Math1 cDNA (pcDNA3.1-Math1) was kindly provided by Dr. Huda Y. Zoghbi (Baylor College of Medicine, Houston, TX, USA). ${ }^{15}$ Full-length Math1 cDNA with target sites of restriction enzymes (Bam $\mathrm{HI}$ and Cla I) was amplified from pcDNA3.1-Math1 by a PCR reaction using the following primers: $5^{\prime}$-CGGGATCCACCATGTCCCGCCTGCTGCATGC-3' and $5^{\prime}$-CCATCGATCTAACT GGCCTCATCAGAGT-3' (underlined portions denote the target site of Bam HI and $\mathrm{Cla}$ I, respectively). The resulting Math1 cDNA was subcloned into Bam $\mathrm{HI}$ and $\mathrm{Cla}$ I sites of the Tet-On gene regulation vector pTRE2pur (pTRE2pur-Math1 plasmid, Supplementary Information, Supplementary Figure S1A).

ES cells. Undifferentiated ES cells (EB3, a kind gift from Dr. Hitoshi Niwa, RIKEN Center for Developmental Biology, Kobe, Japan) were maintained in gelatin-coated dishes without feeder cells in DMEM medium (Sigma, St. Louis, MO, USA) supplemented with 10\% FBS (GIBCO, Invitrogen, Carlsbad, CA, USA), $0.1 \mathrm{mM}$ 2-mercaptoethanol (Sigma), $0.1 \mathrm{mM}$ nonessential amino acids (GIBCO), $1 \mathrm{mM}$ sodium pyruvate (Sigma), and $1000 \mathrm{U} / \mathrm{ml}$ leukemia inhibitory factor (LIF; CHEMICON, Millipore, Billerica, MA, USA). The EB3 cells were sublines derived from E14tg2a ES cells and carried a blasticidin S-resistant selection marker gene driven by the Oct-3/4 promoter (active in undifferentiated state). ${ }^{35}$

Establishment of ES cells carrying tetracycline-inducible Math1. pTet-On (neomycin-resistance gene expression plasmid) was transfected into undifferentiated ES cells (Supplementary Information, Supplementary Figure S1B). The cells were allowed to grow for 2 days, then selected based on their ability to grow in ES medium containing $400 \mu \mathrm{g} / \mathrm{ml} \mathrm{G} 418$. In order to select stable transfectants of pTet-On, neomycin-resistant cells were transiently transfected with pTRE2hyg-Luc (luciferase reporter plasmid). Luciferase-expressing clones, which grew in a Dox-dependent manner, were identified using a luciferase assay system (BD Bioscience), and a single clone expressing high luciferase activity was used for the following transfection of pTRE2pur-Math1 or pTRE2pur (vector alone). After allowing to grow for 2 days, selection was performed based on the ability to grow in ES medium containing $5 \mu \mathrm{g} / \mathrm{ml}$ puromycin and $400 \mu \mathrm{g} / \mathrm{ml} \mathrm{G} 418$. Stable cell lines were then cloned, expanded, and used in culture experiments. The clone transfected with pTRE2pur-Math1 was named Math1-ES.

In vitro HC differentiation procedure. Differentiation of undifferentiated wild-type ES (WT-ES) or Math1-ES cells into HCs was performed using the procedure shown in Figure 2a. Briefly, WT-ES or Math1-ES cells were dissociated by trypsin to culture in hanging drops to form EBs. ${ }^{36}$ The cell density of each drop was 500 cells per $20 \mu$ l of ES cell medium in the absence of LIF (ES-M). After 4 days, EBs in hanging drops were collected and plated in plastic 100-mm gelatincoated dishes (20 EBs per dish) in ES-M with or without Dox and then allowed to attach to form outgrowths. Half of the culture medium was changed to new medium every 2 days during the 2-week EB outgrowth cultures. 

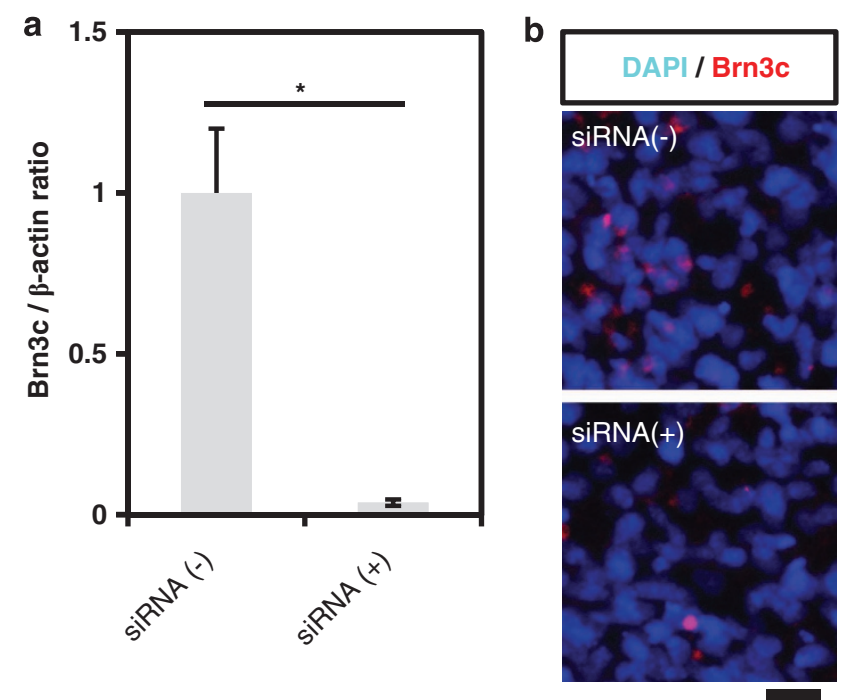

C
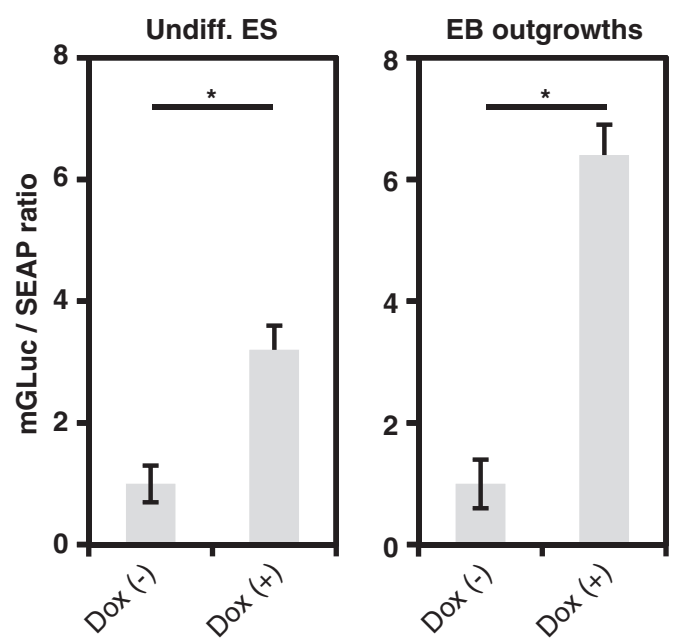

Figure 6 Roles of Math1 in induction of HC-like cells from ES cells. $(\mathbf{a}, \mathbf{b})$ The effects of Math1-specific siRNA on differentiation of HC-like cells from ES cells using the HIST2 method were examined with quantitative PCR. Suppression of Math1 by Math1-specific siRNA inhibited the expression of $B r n 3 c$, an $\mathrm{HC}$-related marker (a), and Brn3c-immunopositive cells were also decreased (b). ${ }^{*} P<0.05$ (c) The promoter activity of Brn3c was examined following transfection of a reporter Brn3c-promoter plasmid. Undifferentiated Math1-ES cells (Undiff. ES) and Math1-ES-derived EB outgrowths cultured with Dox for 14 days (EB outgrowths) were transfected with the pEZX-PG04-Brn3c reporter plasmid and further cultured with or without Dox. After $48 \mathrm{~h}$, cell-free conditioned medium samples were assayed for Gluc and SEAP using a Secrete-Pair Dual Luminescence Assay Kit. The promoter activity of Brn3c was positively regulated in the cultures containing Dox in both undifferentiated ES cells and EB outgrowths. ${ }^{*} P<0.05$. DAPI, 4,6-diamidino-2-phenylindole

RT-PCR. Total RNA was purified using Trizol (Invitrogen) following the protocol of the manufacturer. Extracted RNA was then treated with DNase I (Takara Bio Inc., Otsu, Japan) to remove DNA. After inactivation of DNase I, $1 \mu \mathrm{g}$ of total RNA was synthesized with first-strand cDNA using random primers and reverse transcriptase (M-MLV RT, Promega, Madison, WI, USA) for $1 \mathrm{~h}$ at $37^{\circ} \mathrm{C}$. For PCR analysis, $0.5 \mu \mathrm{g}$ cDNA was used as a template and amplified using the primer sequence shown in Supplementary Information, Supplementary Table S1. The total expressions of Tet-inducible Math1 (exogenous Math1) and endogenous Math1 were detected using 'Math1(T)' primers, while endogenous expression was selectively detected using 'Math1(E)' primers. Math1(E) primers were selected to detect the sequence, which started at position -63 and ended at +415 . The general PCR conditions were $25-30$ cycles at $94^{\circ} \mathrm{C}$ for $2 \mathrm{~min}$,
$94{ }^{\circ} \mathrm{C}$ for $30 \mathrm{~s}, 52-62{ }^{\circ} \mathrm{C}$ for $30 \mathrm{~s}$, and $72{ }^{\circ} \mathrm{C}$ for $1 \mathrm{~min}$, and the products were run on $1.5 \%$ agarose gels.

ALP staining. ALP expression was observed using an Alkaline phosphatase kit (Sigma) according to the manufacturer's protocol. Briefly, cells were washed twice in saline, fixed in fixation solution at room temperature, and washed with water. Dye solution was then added and incubation was performed at room temperature for $30 \mathrm{~min}$. The cells were rinsed well with water 3-4 times and observed under a light microscope.

Immunocytochemistry. Immunofluorescence analysis was performed using a standard protocol. Briefly, cells were fixed in $4 \%$ paraformaldehyde and then cellular membranes were permeabilized with $0.1 \%$ Triton X-100 in PBS containing $1 \%$ BSA (TPBS). The primary antibodies used and dilutions in T-PBS were as follows: anti-Nestin (1: 100, Santa Cruz, Santa Cruz, CA, USA), anti-NF-L (1:100, Santa Cruz), anti-Nkx2.5 (1:200, Abcam, Cambridge, UK), anti-Brn3c (1:400, Covance, Emeryville, CA, USA), anti-Math1 (1:200, CHEMICON), antimyosin6 (1:100, Santa Cruz), anti- $\alpha 9 A$ chR (1:100, Santa Cruz), anti-PDE1C (1:100, Santa Cruz), and anti-GABAa6R (1:100, Santa Cruz). Following incubation overnight at $4{ }^{\circ} \mathrm{C}$ and washing with TPBS three times, AlexaFluor 488- or 546-conjugated anti-goat, anti-rabbit, or anti-mouse secondary antibodies (Molecular Probes, Invitrogen, Carlsbad, CA, USA) were used to detect primary antibodies. All nuclei were stained with DAPI (Dojin, Kumamoto, Japan). After incubation for $1 \mathrm{~h}$ at room temperature and washing with TPBS three times, fluorescence was detected with a laser scanning confocal imaging system (Olympus, Tokyo, Japan), and the images were modified using Photoshop CS (Adobe systems, San Jose, CA, USA) to selectively detect intensely fluorescent cells.

Phalloidin staining. Cells were fixed in $4 \%$ paraformaldehyde, then the cellular membranes were permeabilized with $0.2 \%$ Triton X-100 in PBS. Following incubation for $10 \mathrm{~min}$, the cells were reacted with AlexaFluor 488-labeled phalloidin (diluted 1:40 in PBS, Molecular Probes, Invitrogen) for $20 \mathrm{~min}$ and washed with PBS three times. All nuclei were stained with DAPI and fluorescence was detected using a confocal microscope.

SEM examinations. ES cells underwent differentiation for 18 days and then were re-plated on membrane filters (BD Bioscience). After overnight cultivation, the membranes were washed with PBS and replaced with $5 \%$ glutaraldehyde in $0.1 \mathrm{M}$ sodium cacodylate buffer ( $\mathrm{pH} 7.4$ ) for $45 \mathrm{~min}$. The buffer was removed and the membranes were post-fixed with $1 \%$ osmium tetroxide in buffer $(60 \mathrm{~min})$, washed three times with fresh buffer $(10 \mathrm{~min}$ each) and then distilled water (10 min), and then dehydrated with an ethanol series and HMDS (Polysciences, Warrington, PA, USA) for $5 \mathrm{~min}$. Finally, they were coated with osmium and examined using a SEM (S-5200, HITACHI, Hitachi, Japan).

Dye permeation experiment. Cells were exposed to FM1-43FX $(5 \mu \mathrm{M}$, Invitrogen) for $10 \mathrm{~s}$ at room temperature, washed thoroughly in PBS, then fixed in $2 \%$ PFA, and counterstained with DAPI. ${ }^{29}$ Furthermore, immunostaining for Brn3c was performed following FM1-43FX dye treatment and fixation. Cells in randomly chosen fields $(n=15)$ were examined using confocal microscopy.

siRNA. Cells were transfected with $1 \mu \mathrm{M}$ Math1-specific siRNA (siRNA ID: MS0097825 or MS0097826; Takara Bio Inc.) using Lipofectamine RNAiMAX (Invitrogen), according to the manufacturer's instructions. The effects of Math1 siRNA on expression of Brn3c mRNA were examined with quantitative real-time $\mathrm{PCR}(\mathrm{qPCR}$ ) at $48 \mathrm{~h}$ after transfection.

qPCR analysis. Total RNA $(1 \mu \mathrm{g})$ was extracted from cultured cells using TRIzol reagent (Invitrogen). Reverse transcription and qPCR were performed with a SYBR PrimeScript RT-PCR kit II (Takara Bio Inc.), according to the manufacturer's instructions using primers purchased from Takara Bio Inc. (see Supplementary Table S2). The amount of Brn3c PCR product was calculated relative to the internal control $\beta$-actin and then compared between the experimental and control groups using the $\Delta \Delta \mathrm{CT}$ method.

Reporter assay. Construction of a Brn3c reporter was performed according to the standard cloning protocols. Briefly, the sequence for the mouse Brn3c promoter from -1045 to +46 was amplified from mouse genomic DNA by PCR using gene-specific primers. PCR-amplified CDNA was subcloned into a 

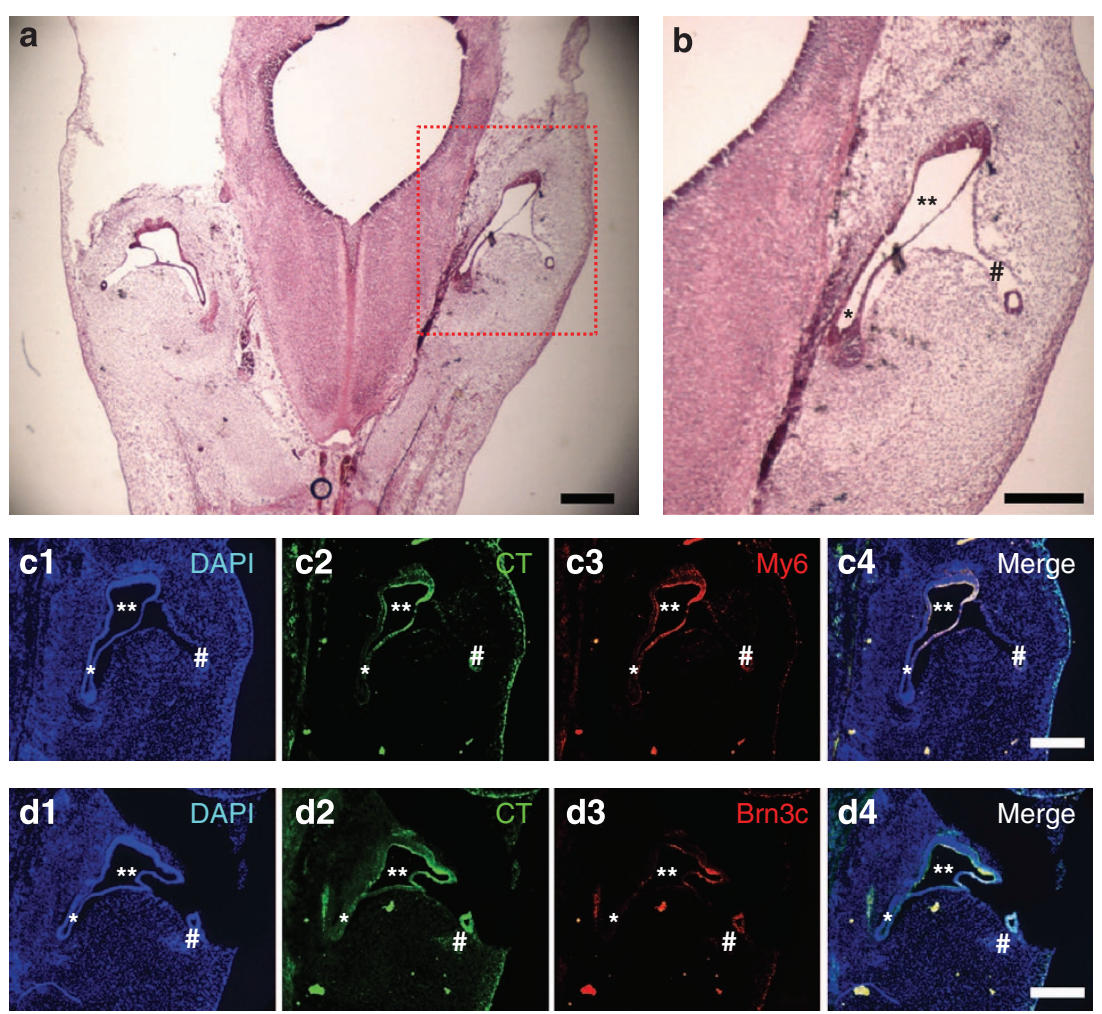

Figure 7 Transplantation of cells from Math1-ES-derived EB outgrowths containing HC-like cells into chick embryos. (a) Graft cells were transplanted into otic vesicles of chicken embryos. Three days after injection, normal morphological processes were confirmed by hematoxylin and eosin staining. Bar $=0.5 \mathrm{~mm}$. (b) Enlarged image of area in (a) marked by dotted line. Bar $=200 \mu \mathrm{m}$. (c and d) Cell tracer-labeled cells (c2, d2, green) were found integrated in the developing inner ear transplanted with cells from Math1-ES-derived EB outgrowths. Most of the integrated cells were immunopositive for myosin6 (c3, red) and Brn3c (d3, red). Bar $=100 \mu \mathrm{m}$. * ${ }^{*}$ chlear duct; **lateral ampulla; \#semicircular canal. DAPI, 4,6-diamidino-2-phenylindole

pEZX-PG04 vector (GeneCopoeia, Inc., Rockville, MD, USA). The plasmid pEZXPG04-Brn3c encodes Gaussia Luciferase (Gluc) under control of the Brn3c-promoter and secreted alkaline phosphatase (SEAP) under control of the CMV promoter.

Cells were plated in 60 -mm dishes $\left(5 \times 10^{5}\right.$ cells/dish) and transfected with a pEZX-PG04-Brn3c plasmid using Lipofectamine 2000 (Invitrogen). Forty-eight hours after transfection, cell-free conditioned medium samples were assayed for Gluc and SEAP using a Secrete-Pair Dual Luminescence Assay Kit (GeneCopoeia Inc.), according to the manufacturer's instructions. Transfection efficiency was normalized to the level of SEAP activity.

Transplantation into chick embryos. Transplantation experiments were performed as previously reported, ${ }^{31}$ with minor modifications. Briefly, undifferentiated ES cells or cells from 14-day Math1-ES-derived EB outgrowths were dissociated with trypsin and labeled with CellTracker Green CMFDA (Molecular Probes). Then, $2 \times 10^{4}$ cells were injected using micro-glass pipettes into the right otic vesicles of day 3 chicken embryos (Hamburger and Hamilton stage 19-20). The left otic vesicles did not receive cell grafting and served as controls for immunohistochemistry examinations. Specimens were fixed for $12 \mathrm{~h}$ in $4 \%$ PFA and embedded in OTC compound. Cross-sections $(6 \mu \mathrm{m})$ were cut using a cryostat. Sections of the inner ear were prepared by axial cutting on day 6.5 of embryonic development. Immunohistochemistry was performed using the protocol described above. The experimental protocols were approved by the Institutional Animal Care and Use Committee of Nara Medical University.

Statistical analysis. The fraction of immunopositive cells among total cells was determined in a double-blind fashion by counting approximately 300 cells in 15 randomly selected microscopic fields per experiment. Three experiments were conducted for each determination, and significance was calculated using the Student's t-test.

\section{Conflict of Interest}

The authors declare no conflict of interest.
Acknowledgements. We thank Dr. H Niwa (CDB RIKEN, Kobe, Japan) for the ES cell lines, Tomoki Nishida (Research Center for Ultrahigh Voltage Electron Microscopy, Osaka University, Ibaraki, Osaka, Japan) for collection and assembly of the electron microscopy data, and Mrs. S Shimada for technical assistance. This research was supported by the 'Nanotechnology Network Project of the Ministry of Education, Culture, Sports, Science and Technology (MEXT), Japan' conducted at the Institute of Scientific and Industrial Research (ISIR)/Research Center for Ultrahigh Voltage Electron Microscopy, Osaka University (Handai Multi-Functional Nanofoundry), and by a Grant-in-Aid for Young Scientists (B) (to YO) and a Grantin-Aid for Scientific Research (B) (to MY) from the Ministry of Education, Culture, Sports, Science and Technology of Japan (KAKENHI).

1. Schacht J. Molecular mechanisms of drug-induced hearing loss. Hear Res 1986; 22: 297-304

2. Hawkins JE Jr., Johnsson LG, Stebbins WC, Moody DB, Coombs SL. Hearing loss and cochlear pathology in monkeys after noise exposure. Acta Otolaryngol 1976; 81: 337-343.

3. Bhave SA, Stone JS, Rubel EW, Coltrera MD. Cell cycle progression in gentamicindamaged avian cochleas. J Neurosci 1995; 15: 4618-4628.

4. Stone JS, Cotanche DA. Hair cell regeneration in the avian auditory epithelium. Int J Dev Biol 2007; 51: 633-647.

5. Stone JS, Rubel EW. Cellular studies of auditory hair cell regeneration in birds. Proc Natl Acad Sci USA 2000; 97: 11714-11721.

6. Chen U, Kosco M. Differentiation of mouse embryonic stem cells in vitro: III. Morphological evaluation of tissues developed after implantation of differentiated mouse embryoid bodies. Dev Dyn 1993; 197: 217-226.

7. Rathjen J, Rathjen PD. Mouse ES cells: experimental exploitation of pluripotent differentiation potential. Curr Opin Genet Dev 2001; 11: 587-594.

8. Takahashi K, Yamanaka S. Induction of pluripotent stem cells from mouse embryonic and adult fibroblast cultures by defined factors. Cell 2006; 126: 663-676.

9. Schwander M, Kachar B, Müller U. Review series: the cell biology of hearing. J Cell Biol 2010; 190: 9-20.

10. Alsina B, Giraldez F, Pujades C. Patterning and cell fate in ear development. Int J Dev Biol 2009; 53: 1503-1513. 
11. Driver EC, Kelley MW. Specification of cell fate in the mammalian cochlea. Birth Defects Res C Embryo Today 2009; 87: 212-221.

12. Kwan T, White PM, Segil N. Development and regeneration of the inner ear. Ann N Y Acad Sci 2009; 1170: 28-33.

13. Lanford PJ, Shailam R, Norton CR, Gridley T, Kelley MW. Expression of Math1 and HES5 in the cochleae of wildtype and Jag2 mutant mice. J Assoc Res Otolaryngol 2000; 1: 161-171.

14. Chen P, Johnson JE, Zoghbi HY, Segil N. The role of Math1 in inner ear development: Uncoupling the establishment of the sensory primordium from hair cell fate determination. Development 2002; 129: 2495-2505.

15. Bermingham NA, Hassan BA, Price SD, Vollrath MA, Ben-Arie N, Eatock RA et al. Math1: an essential gene for the generation of inner ear hair cells. Science 1999; 284: 1837-1841.

16. Zheng JL, Gao WQ. Overexpression of Math1 induces robust production of extra hair cells in postnatal rat inner ears. Nat Neurosci 2000; 3: 580-586.

17. Kawamoto K, Ishimoto S, Minoda R, Brough DE, Raphael Y. Math1 gene transfer generates new cochlear hair cells in mature guinea pigs in vivo. $J$ Neurosci 2003; 23: 4395-4400.

18. Izumikawa M, Minoda R, Kawamoto K, Abrashkin KA, Swiderski DL, Dolan DF et al. Auditory hair cell replacement and hearing improvement by Atoh1 gene therapy in deaf mammals. Nat Med 2005; 11: 271-276.

19. Jeon SJ, Oshima K, Heller S, Edge AS. Bone marrow mesenchymal stem cells are progenitors in vitro for inner ear hair cells. Mol Cell Neurosci 2007; 34: 59-68.

20. Ben-Arie N, Bellen HJ, Armstrong DL, McCall AE, Gordadze PR, Guo Q et al. Math1 is essential for genesis of cerebellar granule neurons. Nature 1997; 390: 169-172.

21. Srivastava R, Kumar M, Peineau S, Csaba Z, Mani S, Gressens $P$ et al. Conditional induction of math1 specifies embryonic stem cells to cerebellar granule neuron lineage and promotes differentiation into mature granule neurons. Stem Cells 2012; 31: 652-665.

22. Gossen M, Bujard H. Efficacy of tetracycline-controlled gene expression is influenced by cell type: commentary. Biotechniques 1995; 19: 213-216; discussion 216-217.

23. Shiroi A, Yoshikawa M, Yokota $H$, Fukui $H$, Ishizaka S, Tatsumi $K$ et al. Identification of insulin-producing cells derived from embryonic stem cells by zinc-chelating dithizone. Stem Cells 2002; 20: 284-292.

24. Mogi A, Ichikawa H, Matsumoto C, Hieda T, Tomotsune D, Sakaki S et al. The method of mouse embryoid body establishment affects structure and developmental gene expression. Tissue Cell 2009; 41: 79-84.

25. Freund $\mathrm{C}$, Ward-van Oostwaard D, Monshouwer-Kloots J, van den Brink S, van Rooijen M, $\mathrm{Xu} \mathrm{X}$ et al. Insulin redirects differentiation from cardiogenic mesoderm and endoderm to neuroectoderm in differentiating human embryonic stem cells. Stem Cells 2008; 26: 724-733.
26. Ishizaka S, Shiroi A, Kanda S, Yoshikawa M, Tsujinoue H, Kuriyama S et al. Development of hepatocytes from ES cells after transfection with the HNF-3beta gene. FASEB J 2002; 16: $1444-1446$.

27. Abdouh A, Despres G, Romand R. Histochemical and scanning electron microscopic studies of supernumerary hair cells in embryonic rat cochlea in vitro. Brain Res 1994; 660: 181-191.

28. Doyle KL, Kazda A, Hort Y, McKay SM, Oleskevich S. Differentiation of adult mouse olfactory precursor cells into hair cells in vitro. Stem Cells 2007; 25: 621-627.

29. Hu Z, Corwin JT. Inner ear hair cells produced in vitro by a mesenchymal-to-epithelial transition. Proc Natl Acad Sci USA 2007; 104: 16675-16680.

30. Furness DN, Richardson GP, Russell IJ. Stereociliary bundle morphology in organotypic cultures of the mouse cochlea. Hear Res 1989; 38: 95-109.

31. Ouji Y, Ishizaka S, Nakamura-Uchiyama F, Yoshikawa M. In vitro differentiation of mouse embryonic stem cells into inner ear hair cell-like cells using stromal cell conditioned medium. Cell Death Dis 2012; 3: e314.

32. Xiang M, Gan L, Li D, Chen ZY, Zhou L, O'Malley BW et al. Essential role of POU-domain factor Brn-3c in auditory and vestibular hair cell development. Proc Natl Acad Sci USA 1997; 94: 9445-9450.

33. Helms AW, Abney AL, Ben-Arie N, Zoghbi HY, Johnson JE. Autoregulation and multiple enhancers control Math1 expression in the developing nervous system. Development 2000; 127: 1185-1196.

34. Woods C, Montcouquiol M, Kelley MW. Math1 regulates development of the sensory epithelium in the mammalian cochlea. Nat Neurosci 2004; 7: 1310-1318.

35. Nishimura F, Yoshikawa M, Kanda S, Nonaka M, Yokota H, Shiroi A et al. Potential use of embryonic stem cells for the treatment of mouse parkinsonian models: improved behavior by transplantation of in vitro differentiated dopaminergic neurons from embryonic stem cells. Stem Cells 2003; 21: 171-180.

36. Keller GM. In vitro differentiation of embryonic stem cells. Curr Opin Cell Biol 1995; 7 : 862-869.

(c) (i) (3) $\odot$ Cell Death and Disease is an open-access journal published by Nature Publishing Group. This work is licensed under a Creative Commons Attribution-NonCommercialNoDerivs 3.0 Unported License. To view a copy of this license, visit http://creativecommons.org/licenses/by-nc-nd/3.0/

Supplementary Information accompanies this paper on Cell Death and Disease website (http://www.nature.com/cddis) 